\title{
Hugoniot Data for Pyrrhotite and the Earth's Core
}

\author{
J. Michael Brown \\ Geophysics Department, Texas A\&M University \\ ThOMAS J. AHrens \\ Seismological Laboratory, California Institute of Technology \\ DenNis L. Shampine \\ Shock-Wave Physics Group, Los Alamos National Laboratory
}

\begin{abstract}
New shock wave Hugoniot data for pyrrhotite $\left(\mathrm{Fe}_{0.9} \mathrm{~S}\right)$ now describe the equation of state to nearly twofold compression at a maximum pressure of $274 \mathrm{GPa}$. A minor discontinuity on the Hugoniot between 100 and $150 \mathrm{GPa}$ is interpreted as the melting transition. While not tightly constrained, the inferred melting point lies below lower-bound temperature estimates based on the Lindemann criterion. The highest-pressure Hugoniot data (representing the melted phase) are used to model the equation of state for liquid iron sulfide. A density for liquid pyrrhotite of $7.80 \pm 0.20 \mathrm{Mg} / \mathrm{m}^{3}$ under core-mantle boundary conditions $(P=135 \mathrm{GPa}, T=4000 \mathrm{~K})$ is calculated. Assuming that sulfur is the primary alloying element in a predominately iron core, the present data are consistent with a homogeneous outer core containing $10 \pm 4 \mathrm{wt} \%$ sulfur.
\end{abstract}

\section{INTRODUCTION}

The use of equation-of-state data from shock wave experiments to constrain the thermal and chemical state of the earth's core has been previously discussed [McQueen and Marsh, 1966; Al'tshuler et al., 1968; Jeanloz, 1979; Ahrens, 1979]. Brown and McQueen [1982] pointed out that constraints based on shock wave data remain weakened by uncertainties which include (1) limited data with nonnegligible experimental errors, (2) uncertain interpretations as to which high-pressure/high-temperature phases are represented by the Hugoniot data, and (3) uncertain values for important auxiliary thermodynamic parameters: namely, the heat capacity and the Gruneisen parameter.

To address the first two points, we have extended the Hugoniot data set for pyrrhotite $\left(\mathrm{Fe}_{0.9} \mathrm{~S}\right)$. Previous data [Ahrens, 1979] characterized the equation of state for this iron sulfide in the pressure regime below $50 \mathrm{GPa}$. However, only two closely spaced data were obtained in the pressure regime near $150 \mathrm{GPa}$, relevant to the core.

As noted by Ahrens [1979], model equations of state under extrapolated conditions of compression are sensitive to the theoretical formalism, i.e., third- or fourth-order Eulerian finite strain versus a linear $U_{s}-U_{p}$ relation. We can now constrain models with data nearly spanning the compression range of interest for the core. Therefore the form of the equation-of-state parameterization becomes less critical.

Mao et al, [1981] presented static compression data for stoichiometric FeS, troilite, to a maximum pressure of 60 $\mathrm{GPa}$. They found FeS in an orthorhombic distorted B1 structure above a pressure of $11.5 \mathrm{GPa}$. From a Murnaghan equation of state a zero-pressure density of $5.6 \mathrm{Mg} / \mathrm{m}^{3}$ and bulk modulus of $137 \pm 18 \mathrm{GPa}$ were determined, assuming a value of 4 for the pressure derivative of the bulk modulus. This represents a volume decrease of $16 \%$ from the low-pressure

Copyright 1984 by the American Geophysical Union.

Paper number 4B0358.

0148-0227/84/004B-0358\$05.00 phase of troilite. The shock wave data for pyrrhotite in the pressure regime between $30 \mathrm{GPa}$ and $120 \mathrm{GPa}$ are basically concordant with the static data. However, analysis of the dynamic data set yields a lower initial bulk modulus (between $101 \mathrm{GPa}$ and $118 \mathrm{GPa}$ ) with a nonconstant pressure derivative of the bulk modulus. With an extended Hugoniot data set we now recognize another small volume change phase transition above $100 \mathrm{GPa}$ for pyrrhotite. Plausibly, this represents the melting transition. Using a new metastable Hugoniot fit to the high-pressure data, previous conclusions regarding the potential sulfur content of the earth's core are strengthened by our current work.

In the analysis presented here, we assume that sulfur is the light element which reduces the density of an iron-dominated mixture in the earth's core. Certainly, scenarios for homogeneous accretion of the earth and subsequent core formation [Stevenson, 1981] as well as with equation-of-state and melting criteria lend support to this sulfur hypothesis. However, arguments based on elemental abundances in the solar system and in $\mathrm{Cl}$ carbonaceous chondrites remain somewhat less concordant.

Ahrens [1979] noted that for a lower bound of 6.5 to 9 weight percent sulfur in the core, the depletion of sulfur in the earth relative to $\mathrm{Cl}$ chondrites is between 1.8 and 3.2. The range depends primarily on the choice of either an olivine or pyroxene stoichiometry for the lower mantle because elemental abundances are referenced to silicon. Using a pyrolite model for the mantle, Ringwood and Keeson [1977] [also Ringwood, 1979, p. 37] showed that a number of volatile elements, in addition to sulfur, are depleted in the earth in relation to chondrites by factors much larger than 3 . They argued that substantial sulfur in the core would implausibly suggest enrichment (less depletion) of sulfur in the earth relative to such less volatile elements as $\mathrm{Cr}, \mathrm{Mn}, \mathrm{Na}, \mathrm{K}, \mathrm{Rb}, \mathrm{F}, \mathrm{Cs}, \mathrm{Zn}$, and $\mathrm{Cl}$.

An alternative perspective is given by Anders and coworkers. Recognizing a variety of chemical processes and multistage thermal history, they use a multiple component model 
TABLE 1. Microprobe Analysis of Pyrrhotite Crystal

\begin{tabular}{cccccc}
\hline & \multicolumn{5}{c}{ Mole Fraction } \\
Aliquot \\
\cline { 2 - 5 } Sample & $\mathrm{Fe}$ & $\mathrm{S}$ & $\mathrm{Ca}$ & $\mathrm{Ni}$ & Formula \\
\hline $\mathrm{F}_{1}$ & 0.53016 & $\mathbf{0 . 4 6 9 7 4}$ & $\ldots$ & $\mathbf{0 . 0 0 0 0 9}$ & $\mathrm{Fe}_{\mathbf{0 . 8 8 6}} \mathrm{S}$ \\
$\mathrm{F}_{2}$ & 0.52811 & $\mathbf{0 . 4 7 1 8 5}$ & $\mathbf{0 . 0 0 0 0 4}$ & $\cdots$ & $\mathrm{Fe}_{\mathbf{0 . 8 9 3}} \mathrm{S}$ \\
$\mathrm{F}_{3}$ & 0.52857 & 0.47136 & $\mathbf{0 . 0 0 0 0 7}$ & $\cdots$ & $\mathrm{Fe}_{\mathbf{0 . 8 9 2}} \mathrm{S}$ \\
\hline
\end{tabular}

Analyst: A. Chodos, California Institute of Technology. Source: Crucero Mine, Anserma, Caladas, Colombia.

for material which accreted to form the earth. Ganapathy and Anders [1974] used an equilibrium condensation sequence for solar gas as the basic framework. They applied known geochemical constraints such as terrestrial abundances for $U, F e$, and a few other elements in a model calculation which estimates fractionation processes. They assume that abundance ratios such as $\mathrm{K} / \mathrm{U}$ and $\mathrm{S} / \mathrm{K}$, which appear invariant in terrestrial rocks, can be used to estimate bulk planet composition. They concluded that the sulfur content of the core should be approximately $5 \mathrm{wt} \%$, based on the same $\mathrm{S} / \mathrm{K}$ ratio for the earth as for the solar system. More recently, Morgan and Anders [1980] noted uncertainties in the understanding of geochemical processes involving sulfur and potassium. They, in fact, accepted the shock wave result of Ahrens [1979] as a reasonable constraint on core sulfur content and listed a com- positional model including $9 \mathrm{wt} \%$ sulfur in the core. They further noted that an equally plausible geochemical solution would be the mixture of $4.5 \mathrm{wt} \%$ sulfur and $4.5 \mathrm{wt} \%$ oxygen.

\section{EXPERIMENTAL TeChNiQue}

Microprobe analyses for three natural pyrrhotite crystals are given in Table 1 . The mean stoichiometry of $\mathrm{Fe}_{0.890 \pm 0.008} \mathrm{~S}$ is similar to that for samples used in the previous study by $A$ hrens [1979]. Using the X ray density composition data for $\mathrm{Fe}_{x} S$ [Robie et al., 1966], a theoretical density of $4.634 \pm 0.014 \mathrm{Mg} / \mathrm{m}^{3}$ is computed for these samples. The average bulk immersion density of $4.610 \pm 0.009 \mathrm{Mg} / \mathrm{m}^{3}$ is $0.5 \%$ less.

Unoriented samples were cut from the three crystals to maximize the volume of usable material. The samples were nominally $12 \mathrm{~mm}$ on a side and 2.4 to $3.1 \mathrm{~mm}$ thick. A 1.5 mm-diameter hole was drilled in the center of each sample. All samples were ground flat to a tolerance of $\pm 0.010 \mathrm{~mm}$ and mounted on 1.6-mm-thick tantalum base plates. Thickness measurements (at specific locations) were repeatable to $\pm 0.001 \mathrm{~mm}$.

The experiments were undertaken using the two-stage light gas gun facility of the Los Alamos National Laboratory. Projectile velocities are typically measured to an accuracy of $0.1 \%$ in the following manner. The projectile passes through two ceramic magnet, multiple-turn coil assemblies at a separation

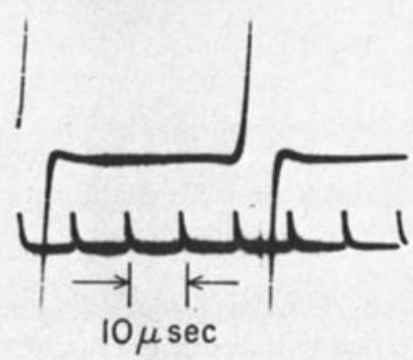

(a)

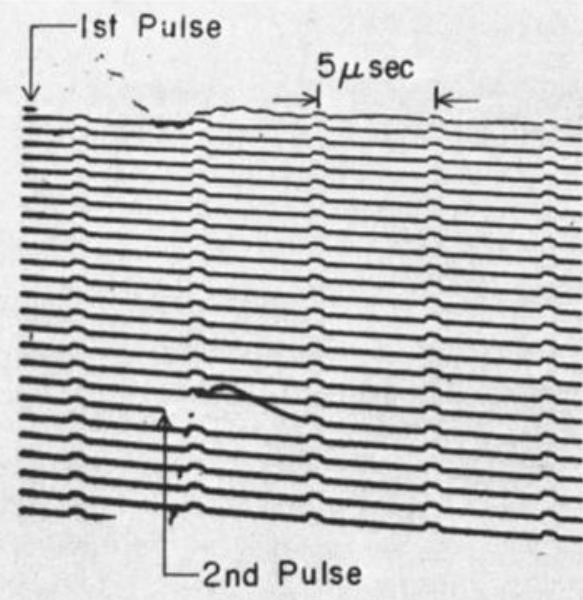

(c)
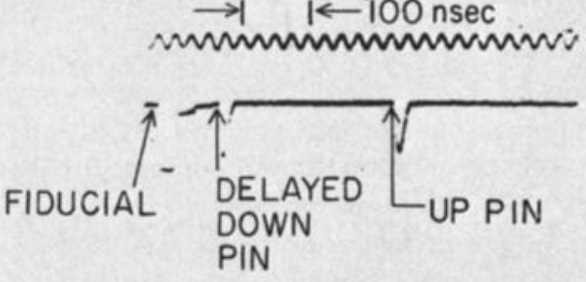

(d)

(b)

Fig. 1. Experimental records for shock wave equation-of-state experiments. (a) Voltage-time record for coil assemblies at a separation of $609 \mathrm{~mm}$. (b) One flash radiograph of the projectile. Note the tantalum flyer molded into a polycarbonate sabot. (c) Raster oscilloscope record of the $X$ ray trigger pulses. The first pulse begins shortly after initiation of the sweep. $(d)$ Pin record for an equation-of-state experiment. 


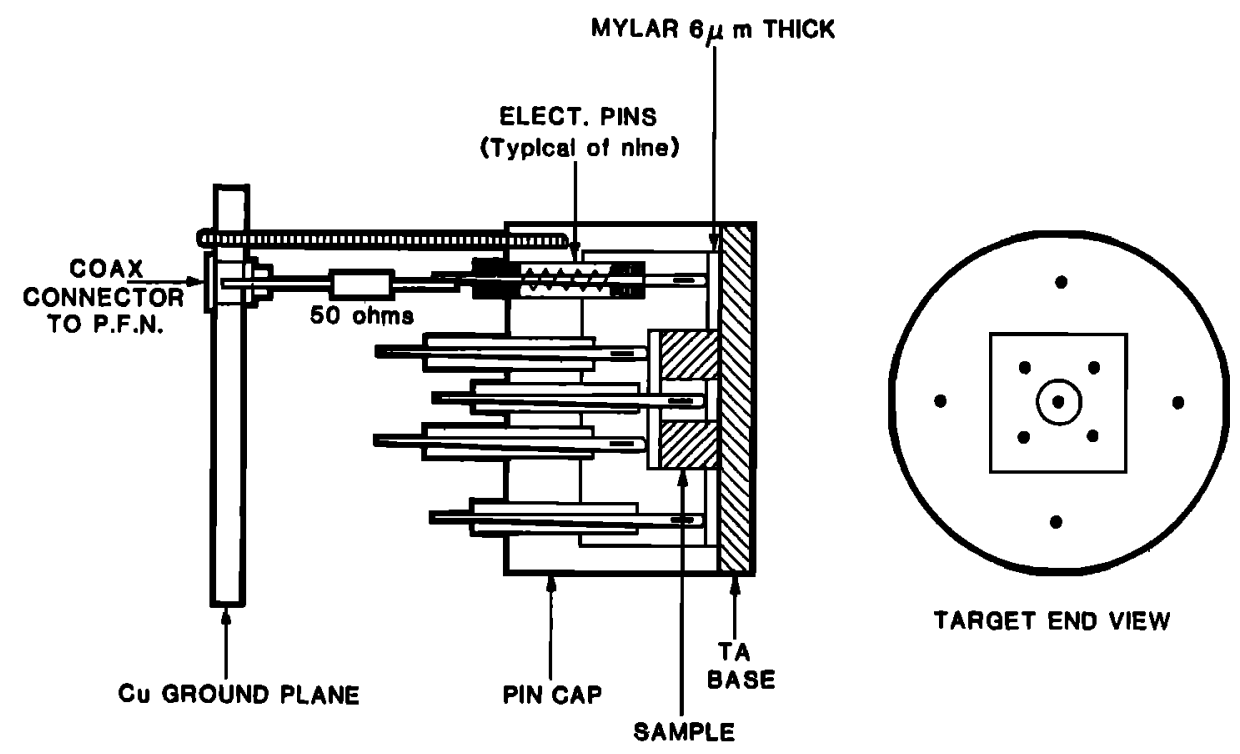

Fig. 2. Equation-of-state target assembly.

of $609 \mathrm{~mm}$. Magnetic flux displacement, caused by the projectile movement, induces an emf (approximately $3 \mathrm{~V}$ across 50 $\Omega$ ) which in turn triggers two flash $X$ ray units as the projectile passes through radiographic fields of view. A voltage-time record from the two coils is shown in Figure $1 a$. One of two flash radiographs is reproduced in Figure $1 b$. The separation of the projectile between the two radiographs can be determined to better than $0.5 \mathrm{~mm}$. The time interval (of the order of $100 \mu \mathrm{s}$ ) between flash radiographs is determined from raster oscilloscope records of the $\mathrm{X}$ ray trigger pulses (Figure $1 c$ ) or with a time interval meter. Reproducibility of timing approaches $10 \mathrm{~ns}$.

Shock wave arrivals on two target levels are detected using electrical pins. Target geometry is illustrated in Figure 2. Electrical pins $(0.41 \mathrm{~mm} \times 30 \mathrm{~mm}$ beading needles) are spring loaded against a $6-\mu \mathrm{m}$ mylar insulating layer. The pins are biased through a capacitor to $150 \mathrm{~V}$ relative to the grounded target assembly.

Shock arrivals at the target surfaces drive the mylar into a conducting state which results in a rapid discharge pulse of the pin bias voltage (rise time $\sim 0.3 \mathrm{~ns}$ ). Four "down" pins (eight on experiment 304) around the periphery of the pyrrhotite samples are used to determine the time of initiation of a shock wave in pyrrhotite. Four "up" pins (eight on experiment 304) atop the pyrrhotite sample mark the shock exit time. A center down pin provides a measure of projectile distortion from planarity.

The first of three arbitrarily chosen down pins to discharge triggers a set of oscilloscopes (typical rise time $\sim 0.3 \mathrm{~ns}$ ). Time fiducials for this initiation point are written on all oscilloscopes. All down pin signals are sent through delay cables (nominal delay $\sim 130 \mathrm{~ns}$, accuracy of delay determination $\sim$ $0.02 \mathrm{~ns}$ ) then multiplexed with the time fiducial generator and an adjoining up pin on each oscilloscope record (Figure 1d). The center pin is recorded on a separate oscilloscope with only the time fiducial. Oscilloscope records of pin shorting times are projected on a digitizing table and read with a reproducibility of better than $1 \mathrm{~ns}$.

Data reduction and error analysis are described by Mitchell and Nellis [1981]. We assumed axially symmetric shock waves characterized by (1) tilt and (2) distortion from planarity of the flyer. The distortion is relatively minor (typically of the order of 1 or $2 \mathrm{~ns}$ ) and was modeled as an axially symmetric parabolic bow. After applying the radially dependent bow correction, we fit all data to a plane wave shock propagation between the down and up pins with three parameters to be determined: (1) angle of impact between the flyer and target, (2) angle between pin 1 and the point of first contact, and (3) transit time through a nominal sample thickness. With eight independent time measurements and three parameters, the problem is overconstrained.

\section{EXPERIMENTAL RESUlts}

Table 2 lists results for six Hugoniot experiments on pyrrhotite. All experiments involved impacting tantalum flyers on tantalum base plates, with the exception of experiment 307 , where a platinum flyer was used. Uncertainties in the projectile velocities are given as the nominal $0.1 \%$ value except for

TABLE 2. Hugoniot Data for Pyrrhotite

\begin{tabular}{ccccccc}
\hline Experiment & $\begin{array}{c}\text { Projectile } \\
\text { Velocity, } \\
\mathrm{km} / \mathrm{s}\end{array}$ & $\begin{array}{c}\text { Initial } \\
\text { Density, } \\
\mathrm{Mg} / \mathrm{m}^{3}\end{array}$ & $\begin{array}{c}\text { Shock } \\
\text { Velocity, } \\
\mathrm{km} / \mathrm{s}\end{array}$ & $\begin{array}{c}\text { Particle } \\
\text { Velocity, } \\
\mathbf{k m} / \mathbf{s}\end{array}$ & $\begin{array}{c}\text { Pressure, } \\
\mathbf{G P a}\end{array}$ & $\begin{array}{c}\text { Density, } \\
\mathbf{M g} / \mathrm{m}^{3}\end{array}$ \\
\hline 308 & $3.983(0.010)$ & $4.611(0.003)$ & $7.341(0.011)$ & $2.816(0.019)$ & $95.3(0.8)$ & $7.480(0.038)$ \\
309 & $4.592(0.005)$ & $4.605(0.003)$ & $7.978(0.009)$ & $3.220(0.019)$ & $118.3(0.8)$ & $7.721(0.037)$ \\
298 & $5.989(0.006)$ & $4.612(0.005)$ & $9.441(0.029)$ & $4.134(0.028)$ & $180.0(1.8)$ & $8.205(0.064)$ \\
299 & $6.528(0.007)$ & $4.617(0.005)$ & $9.951(0.011)$ & $4.489(0.031)$ & $206.3(1.6)$ & $8.412(0.056)$ \\
300 & $6.940(0.007)$ & $4.612(0.005)$ & $10.315(0.018)$ & $4.765(0.034)$ & $226.7(2.0)$ & $8.572(0.066)$ \\
307 & $7.031(0.007)$ & $4.605(0.005)$ & $11.097(0.075)$ & $5.361(0.046)$ & $273.9(4.2)$ & $8.909(0.130)$ \\
\hline
\end{tabular}




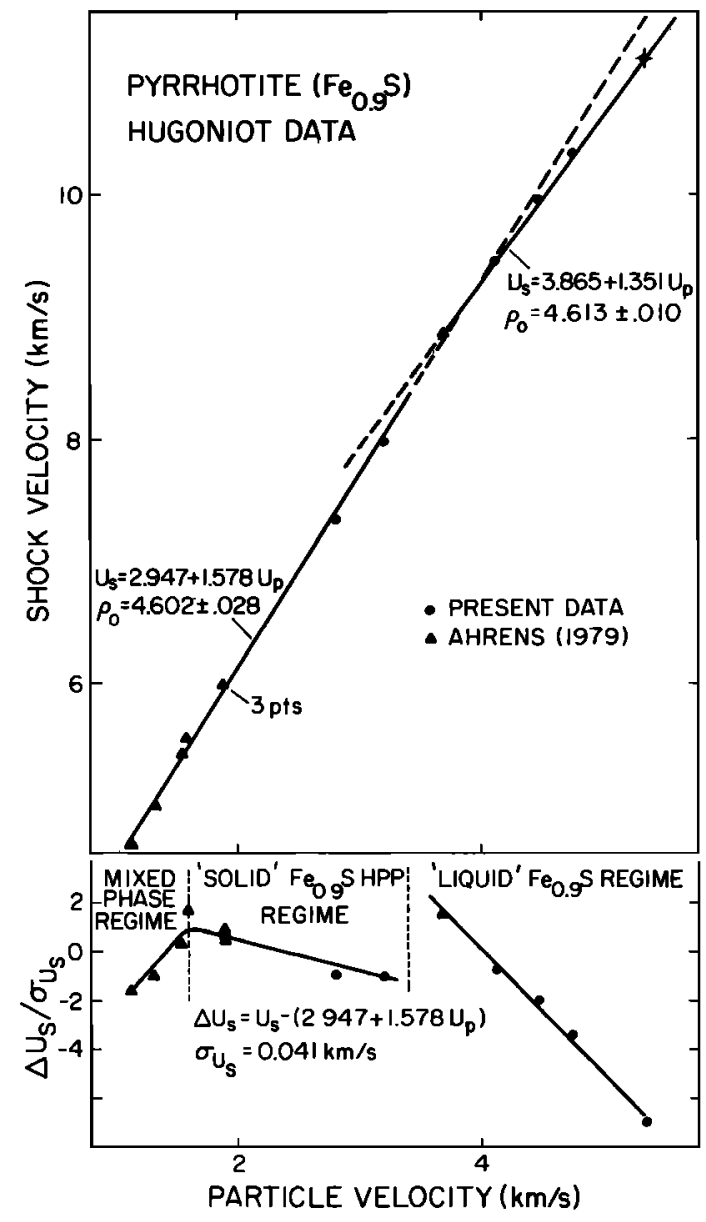

Fig. 3. Pyrrhotite Hugoniol data.

experiment 308. An inferior oscilloscope record limited the accuracy of the timing measurement in that case. Uncertainties listed for shock velocities $\left(U_{s}\right)$ are standard experimental errors [Bevington, 1969]. In all cases, errors were larger than uncertainties in reading oscilloscope records. These deviations are probably associated with either nonaxially symmetric distortion of the flyer or undetected heterogeneity in the natural crystal samples.

Particle velocities $\left(U_{p}\right)$ were calculated using the impedance matching method [McQueen et al., 1970] between the tantalum base and pyrrhotite sample. Hugoniot parameters and uncertainties for tantalum are given by Mitchell and Nellis [1981] and by Marsh [1980] for platinum. The major source of uncertainty in particle velocities for pyrrhotite is the uncertainty in the intercept coeflicient for the linear $U_{s}-U_{p}$ relation for tantalum. Uncertainties in the shock velocities and particle velocities are propagated for determinations of shock pressures and densities using the standard Rankine-Hugoniot relations.
The data for experiment 307 have significantly larger uncertainties. In part these errors were caused by an unusually large projectile tilt. Additionally, experiment 307 required two impedance-matching calculations for both the platinumtantalum interaction and the tantalum-pyrrhotite interaction.

\section{ANALYSIS OF THE DATA}

For the following discussion we include, in addition to the new results, the earlier data [Ahrens, 1979]. Included are the eight experimental points previously used to constrain the high-pressure Hugoniot. The average initial density for those results of $4.603 \pm 0.032 \mathrm{Mg} / \mathrm{m}^{3}$ is $0.15 \%$ less than that for the present experiments. This difference is negligible. We exclude experiment LGG12 since experimental uncertainties were unusually large. Also excluded (by Ahrens [1979] as well) is experiment 241 which deviated substantially from the trend established by other data.

Shock velocity-particle velocity data are plotted in Figure 3. In the lower half of this figure another presentation of the data is shown. Since experimental uncertainties are much smaller than the total range of data, a linear trend through the lower-pressure data has been subtracted from all shock velocities. The resulting deviations from linearity are normalized by an average standard deviation for these experiments. For pyrrhotite, divisions of the data in Figure 3 are evident. The lower-pressure data deviate from a linear trend by less than 2 standard deviations; thus a linear fit may be justified. Note, however, that the systematic behavior of the lower-pressure data is consistent with a nonlinear single phase Hugoniot. Alternatively, Watt and Ahrens [1984] suggest that the mixedphase regime for $\mathrm{Fe}_{0.9} \mathrm{~S}$ may extend to a particle velocity of $1.9 \mathrm{~km} / \mathrm{s}$. Their preferred model, however, has an upper limit of $1.3 \mathrm{~km} / \mathrm{s}$ for the mixed-phase regime.

The five highest-pressure data lie on a strikingly linear trend with a slope less than that for the lower-pressure data. A number of points lead us to the conclusion that these points represent the behavior of liquid iron sulfide. As will be shown later, the discontinuity in Figure 3 at a particle velocity near $3.5 \mathrm{~km} / \mathrm{s}$ falls in a pressure-temperature regime consistent with high-pressure melting behavior. This transition is also associated with a decrease in density of approximately $1 \%$, which is of the right order for a high-pressure melting transition. Also, the slope of the Hugoniot decreases above the discontinuity. This is the expected behavior for a slightly more compressible liquid. Furthermore, preliminary sound velocity data [Brown, 1983] indicate that pyrrhotite is melted above $150 \mathrm{GPa}$ on the Hugoniot.

\section{Equations of State for $\mathrm{FE}_{0}{ }_{9} \mathrm{~S}$}

Direct interpretation of the Hugoniot data for pyrrhotite is complicated by the presence of two-phase transitions. In addition to the melting discontinuity noted in the previous section, Ahrens [1979] found a range of two-wave structure between 5

TABLE 3. Metastable Eulerian Finite Strain (FS) and Shock Wave (SW) Equations of State for High Pressure Phases of Pyrrhotite

\begin{tabular}{llcccccc}
\hline \multicolumn{2}{c}{ Phase } & $\begin{array}{c}\text { Density } \\
\rho_{0}, \mathbf{M g} / \mathrm{m}^{3}\end{array}$ & $\begin{array}{c}\text { Isentropic } \\
\text { Bulk Modulus } \\
K_{0}, \mathrm{GPa}\end{array}$ & $\begin{array}{c}\text { Pressure } \\
\text { Derivative } \\
\text { of } K_{0}, K_{0}\end{array}$ & $\begin{array}{c}\text { Gruneisen } \\
\text { Parameter } \\
\gamma_{0}\end{array}$ & $\begin{array}{c}E_{\text {trans, }} \\
\mathbf{M J} / \mathbf{k g}\end{array}$ & $\begin{array}{c}\text { Standard } \\
\text { Deviation, } \\
\mathrm{GPa}\end{array}$ \\
\hline \multirow{2}{*}{ Solid } & FS & 5.34 & 117.8 & 4.10 & 1.54 & 0.26 & 0.7 \\
& SW & 5.21 & 100.6 & 4.21 & 1.50 & 0.07 & 1.3 \\
Liquid & FS & 5.15 & 98.9 & 4.95 & 1.40 & 0.90 & 1.6 \\
& SW & 5.15 & 124.6 & 4.15 & 1.40 & 0.99 & 0.6 \\
\hline
\end{tabular}




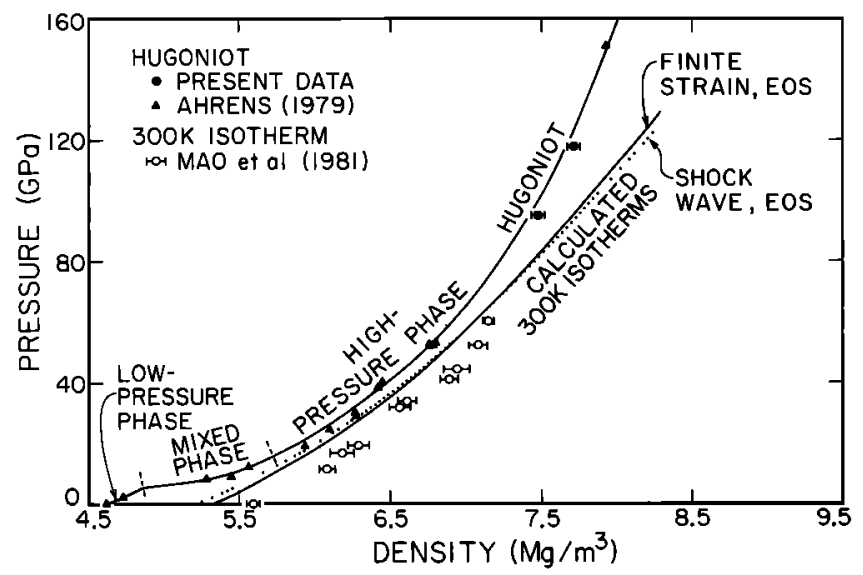

Fig. 4. Shock wave and static compression data for solid iron sulfide and $300 \mathrm{~K}$ isotherms based on the shock wave data.

and $25 \mathrm{GPa}$ associated with the transition of pyrrhotite to the high-pressure solid phase.

We determine metastable Hugoniot and principal isentrope equations of state for both the high-pressure solid and liquid phases of iron sulfide. The model details have been previously discussed [Ahrens, 1979; McQueen et al., 1967].

Equation-of-state parameters for solid and liquid highpressure phases are given in Table 3. Results were determined by least squares fitting of the data in the pressure-volume plane for the finite strain formulation, while the metastable Hugoniots were fit in the shock velocity-particle velocity plane. Because we used experimental results of comparable precision but of different origin, for simplicity, all data were weighted equally. An implicit assumption in the analysis is that $\gamma$, the Gruneisen parameter, is a function of volume only. We further assume that $\rho \gamma$ is constant, where $\rho$ is density. This assumption must be tested in future work.

We note that the number of parameters in these models $\left(\rho_{0}\right.$, $\left.K_{0}, K_{0}{ }^{\prime}, E_{\text {trans }}, \gamma_{0}\right)$ is only slightly less than the number of data. This point alone suggests possible nonuniqueness of models. Furthermore, tradeoffs exists between parameters. The initial density can be adjusted simultaneously with the transition energy and the bulk modulus. Also, the Gruneisen parameter is not well constrained by these data. Caution must be exercised in interpretations based on the zero-pressure parameters, since they represent substantial extrapolation of the data. This is particularly a problem in the case of the high-pressure liquid phase. Note that $K_{0}$ for the shock wave liquid phase fit is implausibly larger than either solid phase fit. Further, the initial density in the finite strain solid phase fit was constrained by the results of Mao et al. [1981]. We conclude that the parameters of Table 3 provide a viable equation of state description only over the range of measurement. Deviations can increase rapidly for extrapolated compression values [see Ahrens, 1979]. The magnitude of additional uncertainties in model values are further discussed below.

The results of Table 3 are not fully thermodynamically consistent in the sense of having rigorously required free energies for two phases to be equal at phase transition points. However, this inconsistency is within errors associated with uncertain values for heat capacity and the Gruneisen parameter as well as with uncertain transition properties such as the transition pressure, the volume change and latent heat of transition.

The value of the Gruneisen parameter remains uncertain. A range of values for $\gamma_{0}$, the Gruneisen parameter at zero pressure, between 1 and 2 is plausible. Larger values are possible for highly anharmonic behavior at high temperature for the higher-pressure phases of pyrrhotite (see, for instance, Jeanloz and Roufosse [1982]). Electronic contributions may also be important but are presently unconstrained by theory and observation. Values listed in Table 3 provide the best fit to the data, but we note that variations in $\gamma_{0}$ within factors of 2 result in negligible model changes.

We assume that lattice heat capacity at constant volume $C_{v}$ is constant and equal to the Dulong-Petit value of $3 k$ per atom, where $k$ is the Boltzmann constant. However, increasingly metallic behavior is expected for pyrrhotite at high pressure. We note that sulfur behaves like a metal above $40 \mathrm{GPa}$ [Dunn and Bundy, 1977; Chhabildas and Ruoff, 1977]. If the electronic contribution to $C_{v}$ for pyrrhotite approaches that found in pure iron, heat capacity may be significantly larger than the Dulong-Petit values under shock wave pressures and temperatures. We model the high-temperature specific heat of pyrrhotite as

$$
C_{v}=3 R+\beta_{0}\left(V / V_{0}\right) T
$$

where $R$ is the gas constant, $\beta_{0}$ is the coefficient of the electronic contribution to the specific heat and the volume compression $\left(V / V_{0}\right)$ provides a variation in the electronic coefficient with compression. Bukowinski [1977] found $\beta_{0}$ equal to $80 \mathrm{~mJ} \mathrm{~kg}^{-1} \mathrm{~K}^{-2}$ using band structure calculations for facecentered cubic iron. In iron the parameter had a volume dependence of $\left(V / V_{0}\right)^{1.5}$. Similar estimates are not available for high-pressure phases of pyrrhotite. We therefore include the range in $\beta_{0}$ from zero to $80 \mathrm{~mJ} \mathrm{~kg} \mathrm{~g}^{-1} \mathrm{~K}^{-2}$ in our estimates of model uncertainties.

In Figure 4 we plot the lower-pressure Hugoniot data for solid pyrrhotite. Also included are the static compression data

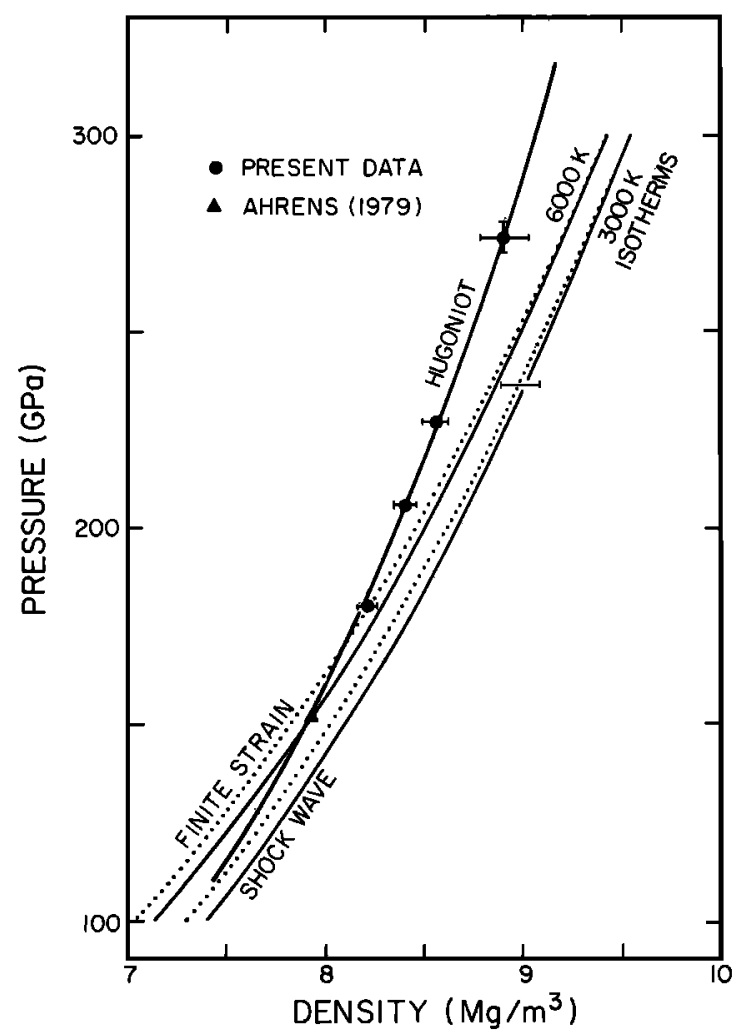

Fig. 5. Shock wave data for liquid pyrrhotite and $3000 \mathrm{~K}$ and $6000 \mathrm{~K}$ isotherms. The error bar on the $3000 \mathrm{~K}$ isotherm indicates the uncertainty associated with uncertain heat capacity and Gruneisen parameter values. 


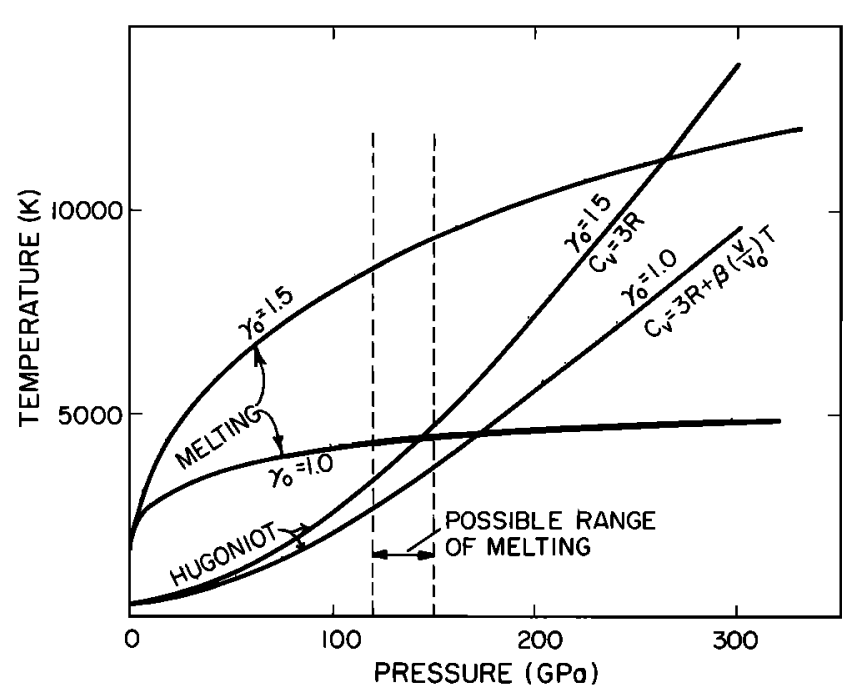

Fig. 6. Hugoniot temperatures and melting phase lines based on the Lindemann melting criterion. Curves based on different values for the Gruneisen parameter and heat capacity are labeled.

for stoichiometric FeS, troilite, by Mao et al. [1981]. Isotherms for $300 \mathrm{~K}$ based on equation-of-state fits to the shock wave data are also plotted. Both shock and static data illustrate the large-volume collapse that pyrrhotite experiences at low pressure, while shock data between $20 \mathrm{GPa}$ and $118 \mathrm{GPa}$ are consistent with the normal compression of a single highpressure phase.

At low pressure, shock heating is modest. Thus parallel trends between static and shock wave data are expected, reflecting only the difference in initial density. However, the highest-pressure static data appear to be anomalously stiff. This implies either that pressures have been overestimated in the static experiments or that shock states are too dense for an applied stress. A similar situation was noted for static and dynamic data for iron [Brown and McQueen, 1982].

In Figure 5 the high-pressure liquid phase data are plotted. Also shown are $3000 \mathrm{~K}$ and $6000 \mathrm{~K}$ isotherms. In each case the finite strain formulation is at most $1 \%$ less dense than the shock wave equation of state. Increasing $\beta_{0}$ from zero to $\mathbf{8 0}$ $\mathrm{mJ} \mathrm{kg} \mathrm{K}^{-2}$ decreases the isothermal density by $1 \%$. A range in $\gamma_{0}$ between 1 and 2 contributes an additional $2 \%$ uncertainty. We conclude that under conditions like those found in the earth's core, a calculated density for liquid $\mathrm{Fe}_{0.9} \mathrm{~S}$ has a probable uncertainty of approximately $\pm 2 \%$.

\section{Melting of $\mathrm{FE}_{0.9} \mathrm{~S}$}

In Figure 6 we plot Hugoniot temperatures and Lindemann estimates for the melting phase boundary:

TABLE 4. Equation of State for $\varepsilon$ Iron and Pyrrhotite Under Core Conditions

\begin{tabular}{|c|c|c|c|c|c|c|}
\hline \multirow{3}{*}{$\begin{array}{l}\text { Pressure, } \\
\text { GPa }\end{array}$} & \multirow{2}{*}{\multicolumn{2}{|c|}{$\begin{array}{c}\varepsilon \text { Iron }^{*} \\
\text { Density, } \mathrm{Mg} / \mathrm{m}^{3}\end{array}$}} & \multicolumn{4}{|c|}{ Pyrrhotite Density, $\mathrm{Mg} / \mathrm{m}^{3}$} \\
\hline & & & \multicolumn{2}{|c|}{ SW } & \multicolumn{2}{|c|}{ FS } \\
\hline & $3000 \mathrm{~K}$ & $5000 \mathrm{~K}$ & $3000 \mathrm{~K}$ & $5000 \mathrm{~K}$ & $3000 \mathrm{~K}$ & $5000 \mathrm{~K}$ \\
\hline 150 & 11.44 & 11.06 & 8.03 & 7.90 & 8.10 & 7.98 \\
\hline 200 & 12.17 & 11.87 & 8.62 & 8.51 & 8.66 & 8.56 \\
\hline 250 & 12.78 & 12.53 & 9.12 & 9.04 & 9.14 & 9.05 \\
\hline 300 & 13.31 & 13.09 & 9.56 & 9.48 & 9.56 & 9.48 \\
\hline
\end{tabular}

*From Brown and McQueen [1982].

SW, shock wave; FS, finite strain.

$$
-\frac{d \ln T_{M}}{d \ln V_{M}}=2 \gamma-2 / 3
$$

where $T_{M}$ is the melting temperature, $V_{M}$ is the volume at melting; and $\gamma$ is the phonon Gruneisen parameter. A range of estimates are shown illustrating the effect of uncertain thermodynamic properties. We assume that $\rho \gamma$ is constant for Hugoniot temperatures and Lindemann calculations. Wolf and Jeanloz [1984] noted the theoretical problems associated with application of the Lindemann criterion to polyatomic systems, and furthermore, they document low-pressure deviations between experiment and theory. However, in the absence of a better model for high-pressure melting, we make the following observations. The uncertainty in the Hugoniot temperature at $150 \mathrm{GPa}$ is approximately $1000 \mathrm{~K}$. Since melting occurs below this pressure, the Lindemann criterion appears to overestimate the melting curve. This is concordant with Lindemann estimates for iron [Brown and McQueen, 1982] and for tantalum [Brown and Shaner, 1984]. Furthermore, assuming that the Lindemann curve provides a qualitatively correct phase boundary at high pressure, the melting point for pyrrhotite at the inner core boundary pressure of $330 \mathrm{GPa}$ is approximately $5000 \mathrm{~K}$. For comparison, the melting point for pure iron is $5800 \pm 500 \mathrm{~K}$ at the same pressure (J. M. Brown and R. G. McQueen, unpublished manuscript, 1984).

\section{The EARTh's Core}

Table 4 lists densities for $\varepsilon$ iron and pyrrhotite under core conditions. The equation of state for $\varepsilon$ iron is given by Brown and McQueen [1982]. We assume that liquid iron is $2 \%$ less dense than $\varepsilon$ iron. This choice is consistent with the iron $\mathrm{Hu}$ goniot of Al'tshuler et al. [1981] with data spanning the melting point for iron. Below melting the Al'tshuler data match predictions based on the $\varepsilon$ iron Hugoniot, while data above the $240-\mathrm{GPa}$ melting point are offset by $0.2 \mathrm{Mg} / \mathrm{m}^{3}$.

A combination of these equation-of-state properties with a geotherm and densities from seismic earth models are used to estimate the mixing ratio of iron plus pyrrhotite necessary to match densities in the liquid outer core [see Ahrens, 1979]. Results are illustrated in Figure 7. Two geotherms were used. Brown and McQueen [1982] estimate a temperature range from $4000 \mathrm{~K}$ to $5300 \mathrm{~K}$ between the core-mantle boundary (CMB) and inner core boundary (ICB). Alternatively, Stacey [1977] estimates a range between $3160 \mathrm{~K}$ and $4200 \mathrm{~K}$. Decreasing temperature increases the apparent sulfur content.

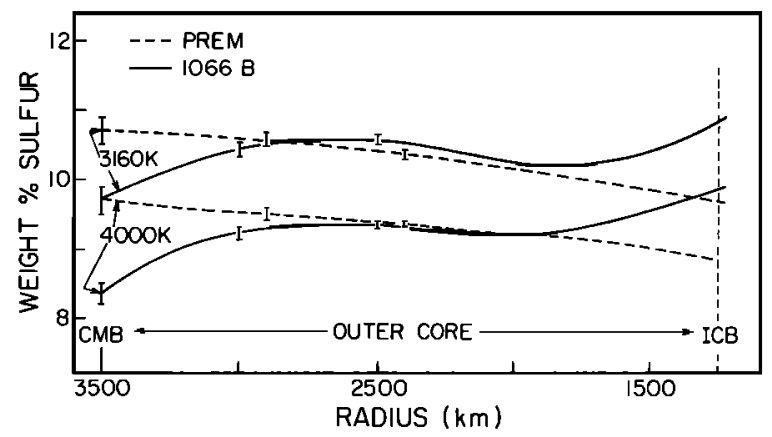

Fig. 7. Weight percent sulfur versus radius calculated from Table 4. Two solid curves are obtained using model 1066B density distribution of Gilbert and Dziewonski [1975], whereas dashed curves correspond to PREM model of Dziewonski and Anderson [1981]. Lowertemperature isentropes centered at $3160 \mathrm{~K}$ at the CMB are from Stacey [1977], while higher-temperature isentropes are from Brown and McQueen [1982]. Bars indicate difference obtained between shock wave and finite strain equation of state. 


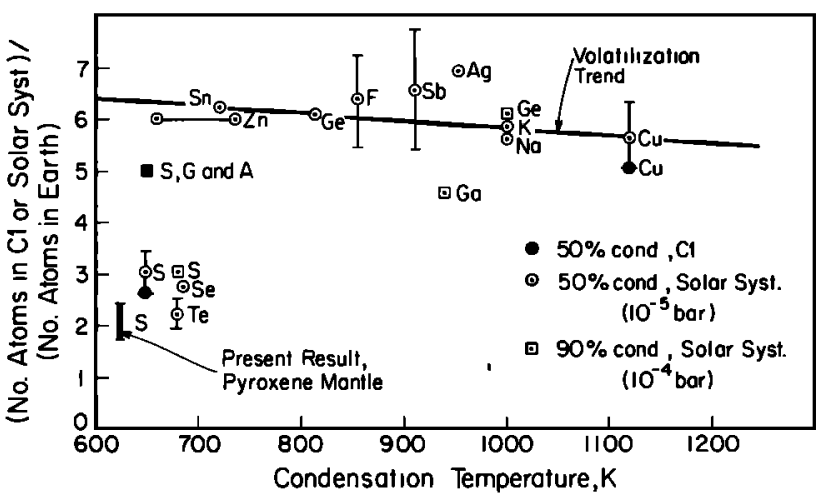

Fig. 8. Depletion of volatile elements in the earth relative to the solar system and $\mathrm{C} 1$ chondrites versus nebular condensation temperature. A separate symbol is shown only if the solar value (open) is significantly greater than the $\mathrm{Cl}$ value. Uncertainties are shown only when larger than $\pm 10 \%$. Condensation temperatures for $50 \%$ condensation at $10^{-5}$ bar $\mathbf{H}_{2}$ pressure are from Ringwood [1979], while the $90 \%$ condensation values at $10^{-4}$ bar are from Grossman and Larimer [1974].

Two seismic earth models were used. Estimates based on the preliminary reference earth model [Dziewonski and Anderson, 1981] indicate a minor decrease in sulfur with depth, while 1066B [Gilbert and Dziewonski, 1975] suggests a slight increase. The effect of equation-of-state parameterization is shown to be minor. However, uncertainties in heat capacity and $\gamma$ contribute an additional 2 wt $\%$ uncertainty in the sulfur content.

In summary, a value of $10 \pm 4 \mathrm{wt} \%$ sulfur is suggested by Figure 7 and our error analysis. The uncertainty is associated with uncertain equations of state, uncertain geotherms, and differing seismic models. We find no compelling evidence for a detectable variation in composition within the outer core. These results strengthen previous conclusions regarding possible sulfur content of the core [Ahrens, 1979; Brown and McQueen, 1982].

\section{The Sulfur Hypothesis}

Using recent estimates for elemental abundances [Anders and Ebihara, 1982], we illustrate arguments for core composition in Figure 8. The abundances in the earth for a selected set of moderately volatile elements relative to $\mathrm{C} 1$ chondrites and the solar system are plotted against their condensation temperature. The majority have remarkably similar depletion factors (between 5 and 7) with anomalies evident for only sulfur and gallium. Sulfur is modestly enriched in the Ganapathy and Anders [1974] model and significantly enriched in the Morgan and Anders [1980] model, in agreement with our current equation-of-state analysis.

For an assumed core chemistry of iron plus sulfur, we conclude that the sulfur content of the core is $10 \pm 4 \mathrm{wt} \%$ based on seismological and equation-of-state data. This result is not in accord with the abundance model based on the mantle composition given by Ringwood [1979]. However, if the earth accreted a solar abundance of sulfur relative to potassium, then a core sulfur content of $5 \mathrm{wt} \%$ is expected [Ganapathy and Anders, 1974]. That result is at the present experimental lower bound. The discrepancy may reflect either uncertainties in the geochemical model [Morgan and Anders, 1980] or uncertainties in the equation-of-state interpretation. Alternatively, other light elements like oxygen [Ringwood, 1979] may, in addition to sulfur, be required to lower densities to those observed in the core. Thus we cannot yet uniquely deter- mine the composition of the core with current experimental and geochemical results.

Acknowledgments. This paper is dedicated to the memory of John C. Jamieson, who was professor of geophysics at the University of Chicago and a frequent visitor at Los Alamos. Jamieson was not only a pioneer in static and dynamic high-pressure research applied to geophysics but also a continuing source of inspiration to our generation of experimentalists. This work was supported in part by the National Science Foundation (grants EAR 8109591 and EAR 8108616) and by the Department of Energy. This work is contribution 31. Geodynamics Program, Texas A\&M University, and contribution 3869, Division of Geological and Planetary Sciences, California Institute of Technology.

\section{REFERENCES}

Ahrens, T. J., Equation of state of iron sulfide and constraints on the sulfur content of the earth, J. Geophys. Res., 84, 985-998, 1979.

Al'tshuler, L. V., G. V. Simakov, and R. F. Trunin, On the composition of the earth's core (Engl. transl.), Izv. Acad. Sci. USSR Phys. Solid Earth, 1, 1-3, 1968.

Al'tshuler, L. V., A. A. Bakanova, I. P. Dudoladov, E. A. Dynin, R. F. Trunin, and B. S. Chekin, Shock adiabatic curves of metals: New data, statistical analysis, and general laws, J. Appl. Mech. Tech. Phys. Engl. Transl., 22, 145-169, 1981.

Anders, E., and M. Ebihara, Solar-system abundances of the elements, Geochim. Cosmochim, Acta, 46, 2363-2380, 1982.

Bevington, P. R., Data Reduction and Error Analysis for the Physical Sciences, 336 pp., McGraw-Hill, New York, 1969.

Brown, J. M., Sound velocities and Gruneisen parameters for troilite and pyrrhotite between $150 \mathrm{GPa}$ and $250 \mathrm{GPa}$ (abstract), Eos Trans. $A G U, 64,848,1983$.

Brown, J. M., and R. G. McQueen, The equation of state for iron and the earth's core, Adv. Earth Planet. Sci., 12, 611-623, 1982.

Brown, J. M., and J. W. Shaner, Rarefaction velocities in shocked tantalum and the high-pressure melting point, in Shock Waves in Condensed Matter, edited by J. Asay, American Physical Society, in press, 1984.

Bukowinski, M. S. T., A theoretical equation of state for the inner core, Phys. Earth Planet. Inter., 14, 333-344, 1977.

Chhabildas, L. C., and A. L. Ruoff, The transition of sulfur to a conducting phase, J. Chem. Phys., 66, 983-985, 1977.

Dunn, K. J., and F. P. Bundy, Electrical behavior of sulfur up to 600 kbar-Metallic state, J. Chem. Phys., 67, 5048-5053, 1977.

Dziewonski, A. M., and D. L. Anderson, Preliminary reference earth model, Phys. Earth Planet. Inter., 25, 297-356, 1981.

Ganapathy, R., and E. Anders, Bulk compositions of the moon and earth, estimated from meteorites, Proc. Lunar Planet. Sci. Conf. 5th, 1181-1206, 1974.

Gilbert, J. F., and A. M. Dziewonski, An application of normal mode theory to the retrieval of structural parameters and source mechanisms from seismic spectra, Philos. Trans. R. Soc. London Ser. A, $278,187-269,1975$.

Grossman, L., and J. W. Larimer, Early chemical history of the solar system, Rev. Geophys. Space Phys., I2, 71-101, 1974.

Jeanloz, R., Properties of iron at high pressure and the state of the core, J. Geophys. Res., 84, 6059-6069, 1979.

Jeanloz, R., and M. Roufosse, Anharmonic properties: Ionic model of the effects of compression and coordination change, J. Geophys. Res., 87, 10,763-10,772, 1982.

Mao, H. K., G. Zou, and P. M. Bell, High-pressure experiments on FeS with bearing on the composition of the earth's core, Year Book Carnegie Inst. Washington, 80, 267-272, 1981.

Marsh, S. P., LASL Shock Hugoniot Data, 658 pp., University of California Press, Los Angeles, 1980.

McQueen, R. G., and S. P. Marsh, Shock wave compression of ironnickel alloys and the earth's core, J. Geophys. Res., 7l, 1751-1756, 1966.

McQueen, R. G., S. P. Marsh, and J. N. Fritz, Hugoniot equation of state of twelve rocks, J. Geophys. Res., 72, 4999-5063, 1967.

McQueen, R. G., S. P. Marsh, J. W. Taylor, J. N. Fritz, and W. J. Carter, The equation of state of solids from shock-wave studies, in High-Velocity Impact Phenomena, edited by R. Kinslow, Academic, New York, 1970.

Mitchell, A. C., and W. J. Nellis, Shock compression of aluminum, copper, and tantalum, J. Appli. Phys., 52, 3363-3374, 1981.

Morgan, J. W., and E. Anders, Chemical composition of earth, Venus, 
and Mercury, Proc. Natl. Acad. Sci. U. S. A., 77, 6973-6977, 1980. Ringwood, A. E., Origin of the Earth and the Moon, 195 pp., SpringerVerlag, New York, 1979.

Ringwood, A. E., and S. Keeson, Siderophile and volatile elements in moon, earth and chondrites, Moon, I6, 425-467, 1977.

Robie, R. A., P. M. Bethke, M. S. Toulmin, and J. L. Edwards, X-ray crystallographic data, densities, and molar volumes of minerals, Mem. Geol. Soc. Am., 97, 29-73, 1966.

Stacey, F. D., A thermal model for the earth, Phys. Earth Planet. Inter., 15, 341-348, 1977.

Stevenson, D. J., Models of the earth's core, Science, 214, 611-619, 1981.

Watt, P. J., and T. J. Ahrens, Shock wave equation of state from the mixed-phase regime, J. Geophys. Res., in press, 1984.
Wolf, G. H., and R. Jeanloz, Lindemann melting law: Anharmonic correction and test of its validity for minerals, J. Geophys. Res., in press, 1984.

T. J. Ahrens, Seismological Laboratory, 252-21, California Institute of Technology, Pasadena, CA 91125.

J. M. Brown, Geophysics Department, Texas A\&M University, College Station, TX 77843.

D. L. Shampine, Shock-Wave Physics Group, Los Alamos National Laboratory, MS970, Los Alamos, NM 87545.

(Received August 15, 1983;

revised February 6, 1984;

accepted February 22, 1984.) 